\title{
Development of Agreeable Models for Army Intelligence, Surveillance and Reconnaissance in Support of Concept Development
}

\author{
$\underline{\text { Ben Pietsch }}^{\mathrm{a}}$, Neville J. Curtis ${ }^{\mathrm{b}}$ and Andrew Coutts ${ }^{\mathrm{a}}$ \\ a Joint and Operations Analysis Division, Defence Science and Technology Group \\ ${ }^{b}$ University of New South Wales in Canberra \\ Email: ben.pietsch@dsto.defence.gov.au
}

\begin{abstract}
Intelligence, Surveillance and Reconnaissance (ISR) is an integral function of the Australian Army, providing information and intelligence to decision makers at all levels. Army's ISR system comprises the equipment, personnel, processes and organisation that conduct collection activities. However, it has been noted that there is a lack of a guiding concept for future development, resulting in unease in acquisition, a lack of integration, and questions over whether the ISR system is being employed as effectively as possible. Accordingly, Army Headquarters (AHQ) has identified a need for a future Land ISR concept, to be supported by analysis from DST Group. This paper focuses on the problem structuring component of that support.

This problem structuring for Army ISR concept development covered the scope, the actors, the current situation, key themes and what the concept is intended to achieve. To do this effectively, it is important to be able to understand, explore and improve the system, and modelling is an important means to represent the system in a way that stakeholders and analysts can agree with, and understand.

Given the nature of the problem, Soft Systems Methodology (SSM) was identified as the most appropriate technique due to its focus on finding out about the problematic situation, which will support development of a range of models. The researchers worked with Army stakeholders to develop a SSM rich picture of the ISR system, an analysis of stakeholder roles, and a set of root definitions that could be developed into activity models. This SSM investigation was supplemented by development of an ISR functional model.
\end{abstract}

The majority of the data collected came from a series of three facilitated workshops with AHQ subject matter experts (SME), where the analyst could ask relevant questions of SME to learn about the situation, and present and refine models. Discussion in these workshops covered the role, scope, actors, key themes and functionality of the Army ISR system. The ISR system rich picture was added to and refined at each workshop to describe the ISR system from capability to effects. Models were used to represent and easily modify what relationship each actor had to the ISR concept, and what they wanted from the concept. A model of ISR functionality was also produced as a framework for analysis of concept options.

Root definitions, or PQR statements, of the form "do P, by Q, in order to achieve R", were developed based on the discussion to capture key points for the concept to address. These statements can also be used as measures of effectiveness for the concept. To be effective the concept must address the PQR statements produced, so that it guides capability decisions, considers future systems, describes how capability provides effects, aligns terminology and addresses key themes.

This report demonstrates the application of SSM as a basis for problem structuring complex military systems, in order to support development of a range of models that can provide guidance to a military concept writing process.

Keywords: Soft systems, concepts, ISR, modelling 


\section{INTRODUCTION}

The aim of this paper is to demonstrate the application of SSM as a useful problem structuring technique for military systems that can support military concept development. SSM underpins the learning process of the analyst and client through structured data capture and the production of models for presentation of information and analysis. The study focused on producing a range of useful models that the analyst and client could agree upon, which represented key aspects of the ISR system and can be used as inputs to concept writing. To do this, an initial understanding of ISR is required.

\section{BACKGROUND}

Conducting Intelligence, Surveillance and Reconnaissance (ISR) is an integral function of the Australian Army and Australian Defence Force (ADF). The Australian Defence Glossary defines ISR as "a collection activity that synchronises and integrates the acquisition, processing and provision of information and intelligence to satisfy a collection requirement" (ADG 2009). ISR is a military function of data collection through a range of means that provides information and intelligence to decision makers at all levels in order to allow them to make the right tactical and operational decisions (Office of ISR Coordination 2007). For the purposes of this study, the client defined the scope of ISR to include the data collection, but exclude fusion and analysis so the 'I' in ISR only covers the collection of intelligence. Thus, ISR fits into the datainformation-knowledge-wisdom (DIKW) hierarchy (Rowley 2007), specifically in the data and information levels of the hierarchy, rather than the knowledge generation aspect of intelligence activities.

Army's ISR system comprises the equipment, personnel, processes and organisation that conduct collection activities. However, Army stakeholders have noted by that there is a lack of a well-defined, Army-wide description of the system, and well-coordinated plan for future development, which, along with the rapid advancements in technology, has resulted in unease in acquisition, a lack of integration of some new elements, and questions over whether the ISR system is being employed as effectively as possible. A number of stovepipe capabilities have emerged, creating integration, interoperability and terminology issues, so Army Headquarters (AHQ) wishes to establish a centralised, function-based vision of ISR capability development. Existing Army ISR documentation is either outdated or limited in scope to a specific sub-unit or capability, without considering future capability. Accordingly, AHQ have identified a need for a future Land ISR Concept document.

The term 'concept' is used broadly in the military context but, in brief, it is a description of a method or scheme for employing specified military capabilities in the achievement of a stated objective or aim (Schmitt 2002; Dortmans et al 2006). This study needed to address the scope, the actors, the current situation, key themes and the overall purpose of the Land ISR Concept. To do this, it is important to be able to understand, explore and improve the system.

\section{METHOD}

This study consisted of problem structuring in support of the ISR concept. This type of situation can be distinguished as either a puzzle, problem or mess (Ackoff 1974; Pidd 2009; NATO 2012a). There is common understanding of the notion of ISR but finer details are not agreed and it is not something that can be reduced to a single measure. Using the checklist from Wijnmalen and Curtis (2013), this situation was determined to be a problem, but one lacking clearly defined objectives from a diverse range of stakeholders. A soft operations research (OR) approach is beneficial as it provides means to exploit human judgement for structuring the problem and in particular, SSM provides a means to define the system from the perspective of multiple stakeholders (Checkland and Scholes 1990). Adams and Meyers (2011) noted that the preferred methodology for framing an unstructured complex systems problem is a rich picture from SSM. Heyer (2004) developed an overview of how soft OR methods including SSM contribute to Defence studies.

The data collection for this study focussed on eliciting expertise from subject matter experts (SME) in order to produce agreed outputs and come to a common understanding using credible OR methods (Rees \& Curtis 2013), so structured workshops with SME were important. The analyst acted in the facilitator role (Franco \& Rouwette 2011) using questions based on SSM techniques (Checkland and Poulter 2010), as well as previous experience and bookwork (Schmitt 2002; Rowley 2007; Office of ISR Coordination 2007; CDG 2010; Headquarters US Army 2010) covering the scope, actors, functions and key themes for the ISR concept.

The first workshop was essentially a scoping workshop to find out what the AHQ client desired for the concept, and introduce the client and SME to the analytical techniques. A strawman system model (rich picture) was presented, to garner immediate feedback to add more detail and make modifications as 
necessary. The second and third workshops followed up the data collected, and presented the next iteration of the ISR system model for feedback; and addressed knowledge gaps identified during analysis of the data.

The following sections discuss the initial scoping of the problem via a rich picture (section 4), the detailing of roles and elements of purposeful activity that define the system (section 4.2), an analysis of roles and motivations (section 4.3), the development of root definitions (section 4.4), a purposeful activity model (section 4.5) and the application of the insights gained from these steps to inform the development of a detailed functional model (section 4.6).

\section{APPLICATION OF SSM}

SSM elements are particularly useful in finding out about the system and are applied to different facets of the problem. Please refer to Checkland and Scholes (1990) for further details on SSM. In this study, the rich picture and functional model describe the system, while the key roles and root definitions focus on requirements for the concept. Concept development is a separate purposeful activity that builds on the knowledge gained in the finding out. The CATWOE step of SSM is informed by the other learning steps.

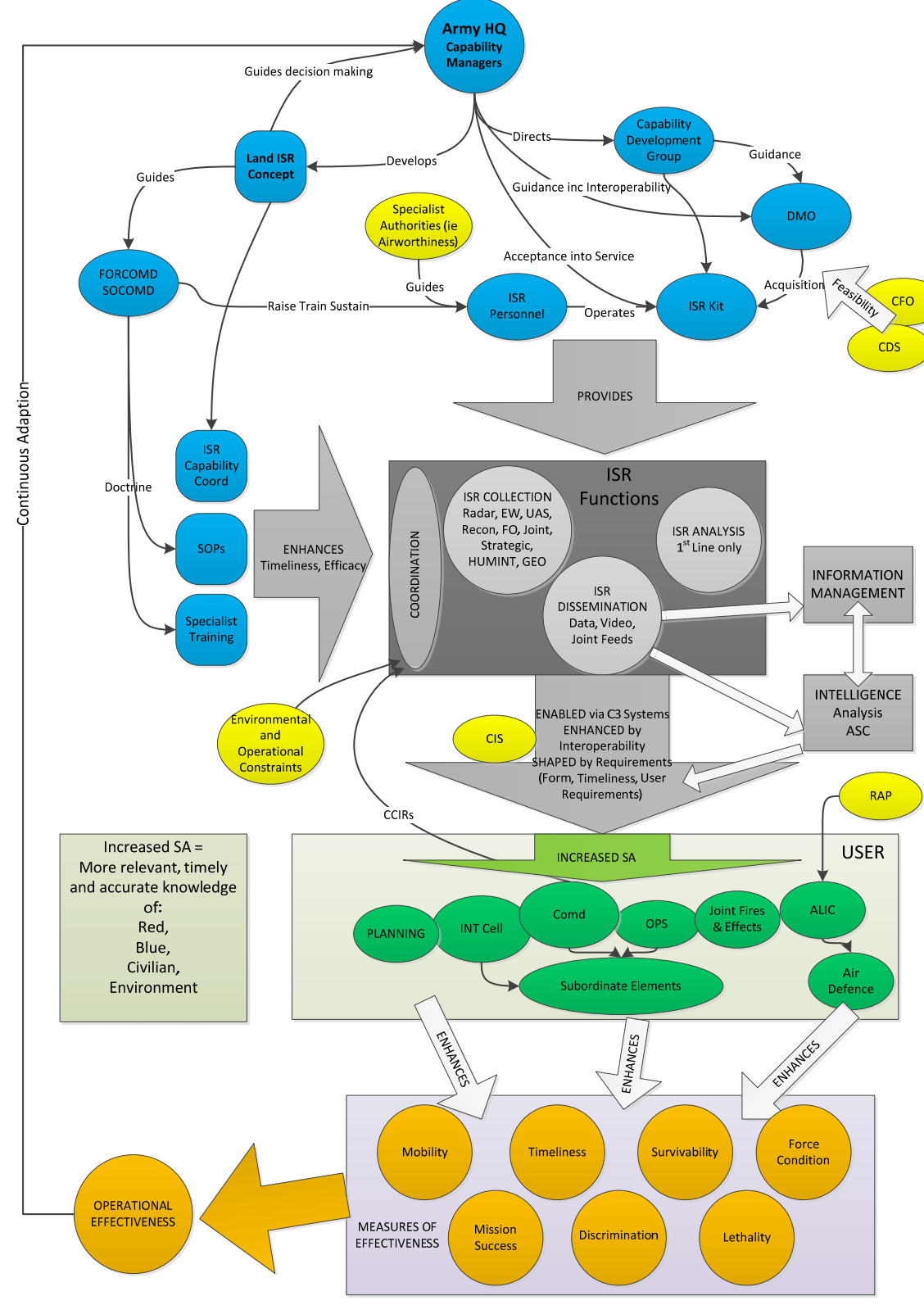

Figure 1. Final iteration of the ISR system rich picture.

\subsection{Rich Picture}

The rich picture was used to work towards a common understanding, through a number of iterations, of the ISR system overview with the client and SME. The initial version was based on a simple framework of the ISR system. The ISR concept needs to describe the ISR capability, but also describe how that capability provides and enhances the ISR functions like collection in order to increase the effectiveness of users.

This framework can also be seen to fit into the PQR (see section 4.3) construct: Do the ISR functions, by employing the ISR capability, in order to achieve enhanced effectiveness for the users.

The flow is shown in Figure 1 with the capability managers at the top providing ISR functions in order to increase the situational awareness of users so that they are more effective. It could be argued that it is more of a diagram than strictly a rich picture (Berg and 
Pooley 2013) which typically depicts the key actors and their perspectives with symbols, sketches or text but with the breadth required, and for ease of manipulation, this was found to be the best way of presenting the analyst's understanding and engaging the SME to revise and agree on the model.

The rich picture was modified based on feedback from the SME workshops, with the final version shown in Figure 1. In addition to the extra details identified, the picture was also useful in demonstrating how the concept not only outlines the provision of ISR capability, such as ISR units, personnel and sensors, but also follows through the flow of how the functions of ISR support military operations.

Note that Figures 1 and 2 contain numerous Defence and Army acronyms which are not defined in this document. The details are outside the scope of this paper, but the structures and flows shown are sufficient to demonstrate how they were used to model the ISR system.

\subsection{CATWOE}

A key step of SSM is to determine system elements which should be considered for any purposeful activity (Checkland and Poulter 2010). CATWOE is a mnemonic of the elements of this situation:

- Customers are the Army ISR stakeholders and users.

- Actors are the capability managers, and the analyst.

- Transformation is the development of the ISR concept which guides capability development.

- Weltanschauung is the worldview described by the root definitions in section 4.3 .

- Owner is the AHQ client.

- Environmental constraints are work effort to produce the final concept, and the uncertainty that is present when trying to predict what is possible in the future due to unknown budgets and advancements in technology.

\subsection{Analysis of Roles and Motivations}

The key roles (Checkland and Poulter 2010) were discussed in the SME workshops in order for the analyst and concept writer to come to a common understanding of what the Concept needs to address from the points of view of the interested parties.

The analyst is the practitioner conducting the study for the AHQ client who caused the

\section{WHAT EVERYONE WANTS FROM THE ISR CONCEPT}
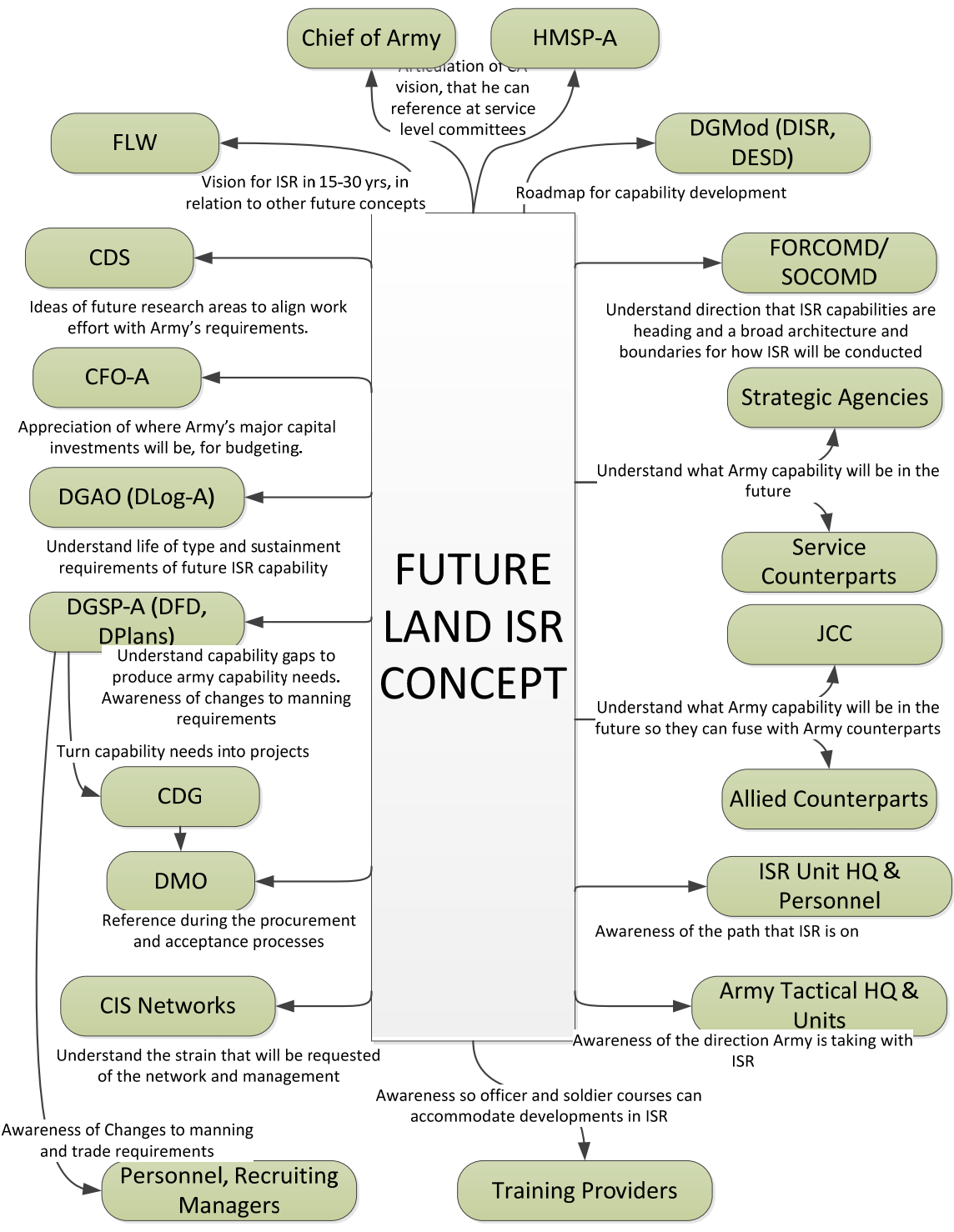

Figure 2. What actors are looking for from the ISR Concept. 
intervention of the development of an ISR concept, while the issue owners were elicited from the data collection. The customers are all of the people or groups (referred to as customers, they are typically Army positions or groups) that are affected by the development of an ISR concept. In addition to categorising the customers and actors, it was important to get an appreciation of their world views based on SME advice, as shown in Figure 2.

\subsection{Root Definitions - PQR Statements}

In SSM, the purposeful activity models are used to represent the system under examination. These models are based on a root definition, in the form of a PQR statement, which is a clear description of the transformation to be modelled. The PQR formula is "do P, by Q, in order to achieve R", which covers the "what, how and why' of the situation (Checkland and Poulter 2010).

The analyst compiled a group of PQR statements based on the key points identified in the workshops, and then refined them based on SME feedback:

- "Articulate a vision of ISR, by describing the ISR capability required, in order to calibrate and justify decision making on ISR capability."

- "Consider timeframes of ISR and linked systems, by performing gap analysis of existing capability life cycles and current projects, in order to ensure the feasibility of the system described in the concept"

- "Centralise ISR capability development and terminology, by producing an Army-wide concept, in order to eliminate stovepipes and ISR integration problems across different Army units."

- "Synchronise employment of ISR assets, by providing awareness of available feeds, in order to achieve better decision making.

- "Ensure effectiveness of sensors links to command and control (C2) structures, by procuring interoperable systems, to generate situational awareness."

- "Outline how ISR capability contributes to operational outcomes, by describing the links between the capability and ISR functions and then how the functions link to operational effectiveness, in order to capture the 'so what?' of ISR capability."

- "Increase persistent surveillance capability beyond the limitations of human endurance, by greater use of robotics or remotely operated systems' sensors, in order to achieve enhanced situational awareness."

- "Enhance discrimination of potential targets, by increased use of non-intrusive biometric capability, in order to mitigate the effects of a crowded, complex environment."

- "Protect ISR collectors, by increased use of robotics or remotely operated system sensors, in order to mitigate the risks of an increasingly lethal environment."

The development of these helped facilitate a shared understanding of the key issues for the concept to address, and helped the SME to clarify the reasons behind their thoughts on the situation. It was also noted by the SME that these statements could be applied to measure the effectiveness of the concept. To be effective the concept must address the PQR statements produced, so that it guides capability decisions, considers future systems, describes how capability provides effects, aligns terminology and addresses key themes.

\subsection{Purposeful Activity Model}

The PQR statement "Outline how ISR capability contributes to operational outcomes, by describing the links between the capability and ISR functions and then how the functions link to operational effectiveness, in order to capture the 'so what?' of ISR capability" is a key narrative of the ISR system. ISR functionality can be can be represented by a model of the flow of information through the ISR system in support of commander decision making. This will help to structure investigations into the effectiveness of the ISR system described by the concept, by outlining how effectively it performs collection and how well that supports commander decision making. The purposeful activity model is shown in Figure 3, and uses the form defined by SSM (Checkland and Scholes 1990).

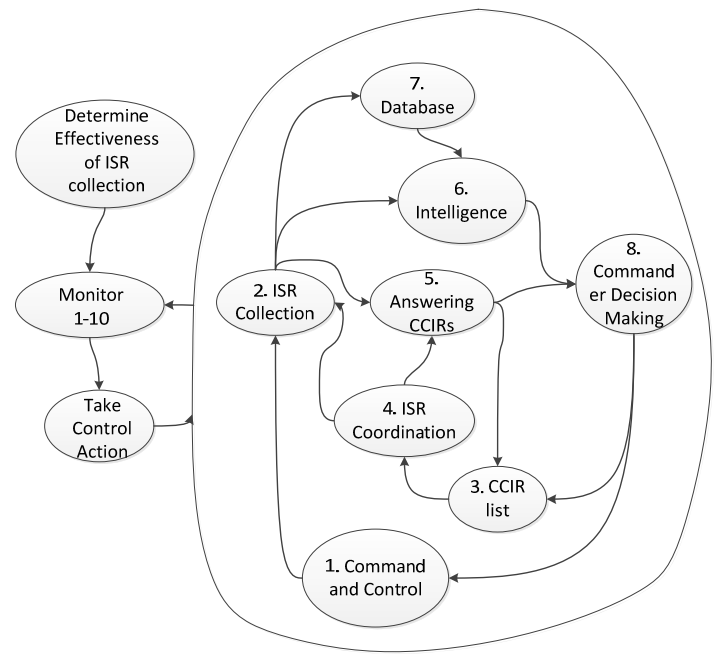

Figure 3. SSM purposeful activity model of ISR functionality. 


\subsection{ISR Functional Model}

Figure 4 presents a detailed ISR functional model, which is based on: existing models (Boyd 1996; Grant and Kooter; 2005; Headquarters US Army 2010); the basic SSM activity model (Figure 3) and the data captured from SME. ISR collectors are coordinated by the Collection Manager in the Intelligence (Int) cell. The crosses in the diagram indicate that constraints can prevent collection. This is the first step in measuring the effectiveness of means to overcome issues in collection. Data from ISR collectors goes directly to the Int cell, fusion and analysis elements, or into a database/archive, and a well-defined procedure for this data management was identified as a key theme for the concept. Manoeuvre elements without a direct link to the Int cell provide ISR in the form of tactical reports which go up the chain of command to reach the Int cell. The Int cell is responsible for passing on the most important and relevant intelligence to the Commander to aid decision-making.

The C2 part of the model is made up of two distinct streams: the tactical (labelled ORDERS) for the ISR collectors from the commander, which are managed by the Operations Cell; and the guidance from the Commander about what information is required, labelled as Commander's Critical Information Requirements (CCIRs).

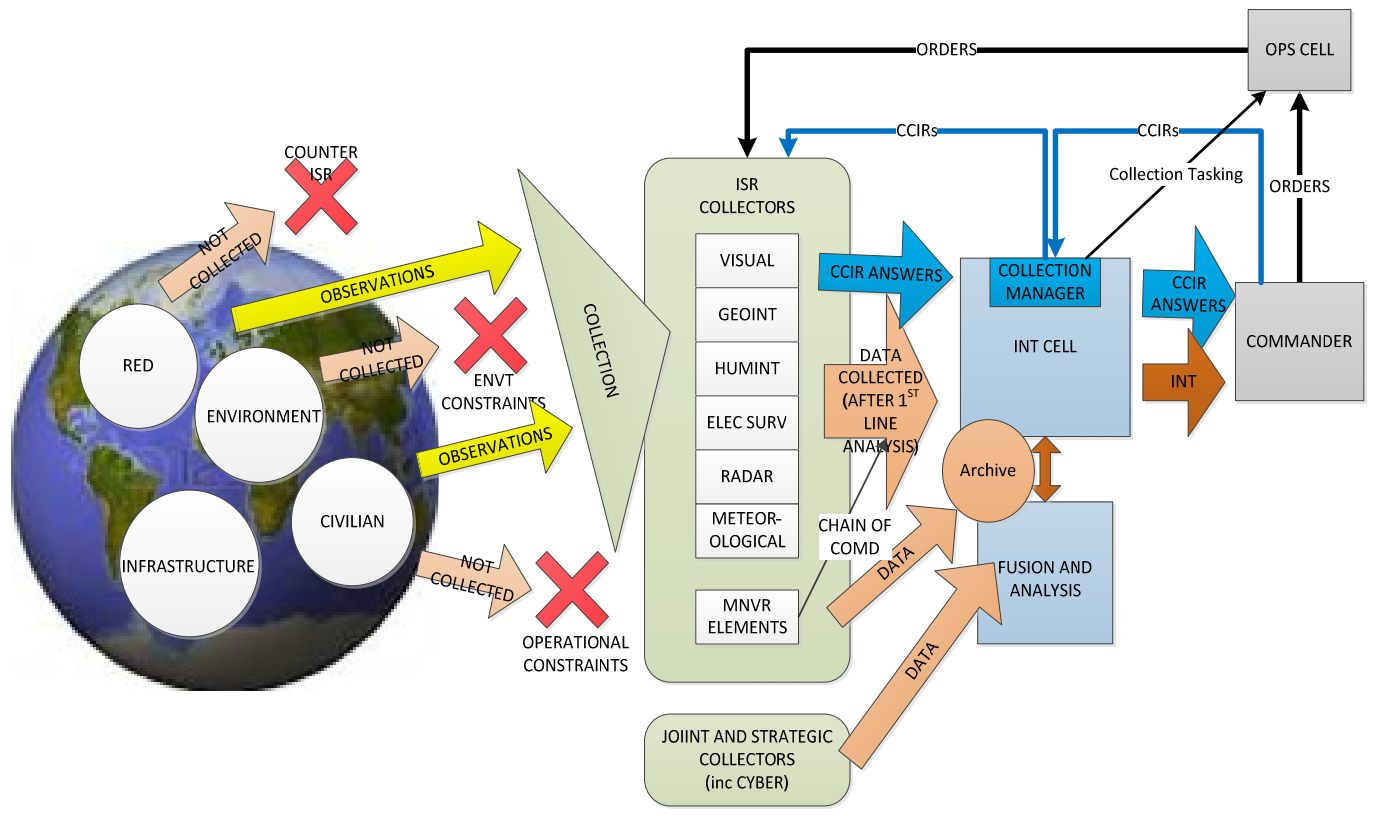

Figure 4. Detailed ISR functional model.

This functional model is designed to provide a framework that can be used to structure investigation into the effects that changes to capability or processes, have on the flow of information. Thus the model is a tool that can be used to support monitoring the effectiveness of the ISR system, which adds detail to the basic structure of the SSM purposeful activity model. It also aids in understanding the functions of the ISR system as a whole, which is useful to help analysts and clients come to a shared understanding of the system. This model could be developed into a quantitative model for evaluation of different concept solutions that would add analytical rigour to the qualitative method, and be more likely to lead to an implementable solution.

\section{CONCLUSIONS}

This paper has demonstrated that SSM can be used as a basis for problem structuring within complex military systems in order to support the development of a military concept. Specific elements of SSM generated in this study - including key aspirational statements for the ISR function and models describing the ISR system - have directly informed Army concept writers. The utilisation of an accepted method (SSM) as a basis for the study increased their confidence in the enquiry process and in the resulting products and insights produced (NATO 2012b). Finally, the authors received positive feedback from AHQ on the benefits of the study and the utility of the approach in supporting concept development more generally. 
Pietsch et al., Development of Agreeable Models for Army ISR in Support of Concept Development

\section{REFERENCES}

Ackoff, R.L. (1974). Redesigning the future. John Wiley \& Sons, New York.

Adams, K. M., \& Meyers, T. J. (2011). Perspective 1 of the SoSE methodology: framing the system under study. International Journal of System of Systems Engineering, 2(2-3), 163-192.

ADG. (2009) Australian Defence Glossary. Available from: http://adg.eas.defence.mil.au

Berg, T., \& Pooley, R. (2013). Rich pictures: Collaborative communication through icons. Systemic Practice and Action Research, 26(4), 361-376.

Boyd, J.R. (1996) The essence of winning and losing. Unpublished lecture notes, 1996.

Checkland, P. and J. Poulter. (2010). Soft Systems Methodology, in Systems Approaches to Managing Change: A Practical Guide. M. Reynolds and S. Holwell, Editors. The Open University: London.

Checkland, P. and J. Scholes. (1990). Soft systems methodology in practice. J Wiley Chichester UK.

Dortmans, P. J., N. J. Curtis, and N. Tri. (2006) An analytical approach for constructing and measuring concepts. Journal of the Operational Research Society 57.8.

Franco, L. A., \& Rouwette, E. A. (2011). Decision development in facilitated modelling workshops. European Journal of Operational Research, 212(1), 164-178..

Grant, T. and B. Kooter. (2005). Comparing OODA \& other models as Operational View C2 Architecture Topic: C4ISR/C2 Architecture. ICCRTS2005, June.

Headquarters US Army. (2010) Field Manual 2-0 Intelligence. Available from: http://fas.org/irp/doddir/army/fm2-0.pdf [accessed 9 October 2014].

Heyer (2004). Understanding Soft Operations Research: The methods, their application and its future in the Defence setting. Defence Science and Technology Organisation DSTO-GD-0411.

NATO. (2012a). NATO Guide for Judgement-Based Operational Analysis in Defence Decision Making (Analyst Volume). RTO TR-SAS-087.

NATO. (2012b). NATO Guide for Judgement-Based Operational Analysis in Defence Decision Making (Client Volume). RTO TR-SAS-087.

Office of ISR Coordination. (2007). Defence ISR Roadmap. Intelligence Security and International Policy, Department of Defence.

Pickburn, G. (2001). Guidance on the use of Subjective Methods in Operational Analysis, D.G.S.A., Editor. 2001, UK Ministry of Defence.

Pidd, M. Tools for thinking. 2009: Wiley Chichester, UK.

Rees, L. M. L., \& Curtis, N. J. (2013). Framing elements for data collection in army field environmentsproblem structuring for acquisition of the right data right. ASOR Conference proceedings.

Rowley, J.E. (2007) The wisdom hierarchy: representations of the DIKW hierarchy. Journal of Information Science.

Schmitt, J.F. (2002). A Practical Guide for Developing and Writing Military Concepts, Defense Adaptive Red Team Working Paper. Hicks and Associates Inc: McLean, Virginia.

Wijnmalen, D.J.D. and N.J. Curtis. (2013). A code of best practice for judgement-based operational research. OR Insight 26: 291-308. 